\title{
ANALYSIS OF PROPORTIONAL MACHINE ALLOCATION SCHEME IN A DETERMINISTIC REENTRANT LINE ${ }^{+}$
}

\author{
Shi-Chung Chang ${ }^{l *}$, Wen-Lin.Jan ${ }^{1}$, Tza-Huei Wang ${ }^{2}$ and Cheng-Shang Chang ${ }^{3}$ \\ 1 Room 245, Dept. of Electrical Engineering, National Taiwan University, Taipei, Taiwan, R.O.C. \\ e-mail : scchang@ac.ee.ntu.edu.tw \\ 2 Dept. of Mechanical Engineering, National Taiwan University, Taipei, Taiwan, R.O.C. \\ 3 Dept. of Electrical Engineering, National Tsin Hua University, Hsin-Chu, Taiwan, R.O.C.
}

\begin{abstract}
This paper analyzes the properties of a proportional machine allocation scheme in a reentrant production line of one product and one machine group. The scheme allocates machines to each production stage proportional to the workload of the stage. It is proved that given initial work-in-process (WIP) distribution and a constant work release rate in the capacity of the line, there exists a unique, full machine capacity allocation for the line; when the proportional machine allocation scheme is repetitively applied over time, the whole reentrant line is eventually balanced. These results obtained under such a simplified scenario also provide some insights to the dynamics and resource allocation problem of a reentrant line.
\end{abstract}

\section{Introduction}

Integrated circuit (IC) wafer fabrication is a business well known for its high capital investment and fierce competition. Investments on highly delicate fabrication equipments constitute a major portion of the capital investment. Effective utilization of these expensive equipments for high productivity is very important to the competitiveness of IC wafer fabrication business.

The fabrication process of each type of wafers may require about 100 fabrication stages; each stage consists of a few fabrication steps and the whole process involves tens of machines. As a type of wafers have quite a few (10-30 or so) layers of fabrication and stages between two layers of a wafer bear some basic similarity, the production flow of each type of wafers may revisit the similar sequence of machine groups from layer to layer in its fabrication process [AtD86]. Owing to such a ręentrant nature, wafers of different part types as well as wafers of the same type but at different stages of fabrication may compete for the finite capacity of a machine group.

+ This work was supported in part by the National Science Council of the Republic of China under Grant NSC 84-2212-E-002-075 and by Taiwan Semiconductor Manufacturing Co. under contact $82-\mathrm{S}-40$

* The authors would like to thank Mr. C. K. Wang, Mr. K. C. Lin, Mr. W. H. Tai, Ms. I. C. Hsu and Mr. S. R. Huang of TSMC for their support and invaluable comments.

$0-7803-2535-4 / 95 \$ 4.00 @ 1995$ IEEE 
How to allocate machine capacities to various stages sharing the same machine group to achieve a steady fab output, a smooth wafer flow at each fabrication stage and a short production cycle time is a very significant and challenging issue for production control of such a system. Recently, there has been growth in research on loading, scheduling and dispatching of IC wafer fabrication [BiT88, LoG88, Wei88, BSG90, LuK91, CFY92, LRK93]. Among them, [Kum93, LRK93, PeK94] performed some theoretic as well as simulation study of reentrant lines. Chang et al. [CLP92] developed an effective algorithm based on a deterministic problem formulation for daily production target generation and machine allocation and achieved successful implementation in an IC wafer fabrication plant. Their algorithm adopts an iterative and proportional resource allocation approach that combines deterministic queueing analysis and some empirical rules. In spite of these existing results, there is yet much to be learned about the dynamics and the control of complex reentrant lines.

Motivated by successful field application results of [CLP92], we analyze in this paper the properties of a proportional machine allocation scheme for a reentrant line. A simplified scenario is considered, where there is only one product type and one machine group, the processing times are deterministic but different over different stages, and machines do not fail. The proportional allocation scheme allocates machine capacity to individual production stages proportional to the total workload (existing inventory and anticipated flow-in from the up stream) of each stage. We show that such a simple scheme fully utilizes the available machines and when it is repetitively applied period by period, the reentrant line can be balanced while maintaining maximum throughput. Although much simplified without considering many features such as machine failures, batching and setup, our analysis does provide some insights basic to both the properties of machine allocation and the dynamics of reentrant lines.

\section{Problem Description}

Consider a reentrant line that produces a single type of products as shown in Figure 2.1. There are J stages in the production process flow, sharing one group of $M$ homogeneous machines with an infinite input buffer for each stage. It is assumed that these machines do not fail. Wafers are released into the first buffer for processing and finished wafers come out of the Jth stage. Let us define some notations for constructing a discrete time model of the work flow dynamics in the reentrant line as follows.

\section{Notations:}

$J:$ total number of stages in the production process flow;

$j$ : stage index, $\mathrm{j}=1, \ldots, \mathrm{J}$;

$n:$ time period index, $\mathrm{n}=0,1,2, \ldots$;

$M:$ total number of machines in the machine group;

$T$ : length of a time period, (for example, one day);

$r_{0}(n)$ : the amount of wafers released into the line at the beginning of the nth period;

$\tau_{j}$ : the processing time of a wafer by a machine at the jth stage;

$w_{j}(n)$ : WIP level of the jth buffer at the beginning of the nth time period;

$m_{j}(n)$ : number of machines allocated to the jth stage during the nth time period;

$r_{j}(n)$ : number of wafers processed at the jth stage during the $n$th period. 


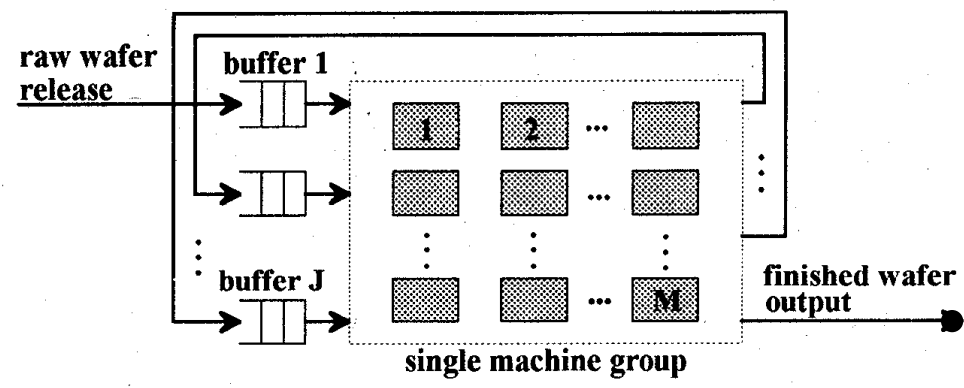

Figure 2.1 A reentrant line of single product type and single machine group

For a deterministic reentrant line described above, the processing time requirement of each wafer is equal to $\sum_{j=1}^{J} \tau_{j}$. Thus, the maximum long term average output is $(M T) /\left(\sum_{j=1}^{J} \tau_{j}\right)$, which is considered as the output capacity of the line. In order to operate the line in full capacity, we set the amount of wafers released into the first buffer at the beginning of each period as

$$
r_{0}(n)=r_{0}=(M T) /\left(\sum_{j=1}^{J} \tau_{j}\right)
$$

As all the $\mathrm{J}$ stages share the $\mathrm{M}$ machines, a policy is needed for allocating machines to process wafers at different stages such that the reentrant line not only generates the maximum long term average output but also reaches the situation of line balancing, which is formally defined as follows.

\section{Definition 2.1 : (Line Balance)}

When the outputs of all stages are equal, then the reentrant line reaches line balance.

In this paper, we study an intuitive machine allocation rule, where the machine capacity allocated to a stage is proportional to and limited by the workload of the existing inventory plus anticipated flow-in of the stage, i.e.

$$
m_{j}(n)=\min \left\{\frac{\left[w_{j}(n)+r_{j}(n)\right] \tau_{j}}{T}, \frac{\left[w_{j}(n)+r_{j-1}(n)\right] \tau_{j}}{\sum_{i=1}^{J}\left[w_{i}(n)+r_{i-1}(n)\right] \tau_{i}} M\right\}
$$

With machine allocation $m_{j}(n)$, the output target of the jth stage is

$$
r_{j}(n)=m_{j}(n) \frac{T}{\tau_{j}}=\min \left[w_{j}(n)+r_{j-1}(n), \frac{\left[w_{j}(n)+r_{j-1}(n)\right]}{\sum_{i=1}^{J}\left[w_{i}(n)+r_{i-1}(n)\right] \tau_{i}} M T\right] \quad \text { for } j=1, \ldots, J .
$$

Equation (2.3) represents the dynamics of part flow under the proportional machine allocation scheme. Note that the number of wafers that can be processed is determined by not only the available machine capacity but also the available WIP level.

\section{Uniqueness of Allocation}

In this section, we show that there exists a unique solution $\left\{r_{j}^{*}(n), j=1, \ldots, J\right\}$ of the nonlinear equation (2.3) under the constant release rate $r_{0}$ for an arbitrarily given WIP distribution $\left\{w_{j}(n), j=1, \ldots, J\right\}$, which is equivalent to the existence of a unique machine allocation $\left\{m_{j}^{*}(n), j=1, \ldots, J\right\}$ for equation (2.2). 
Note that the minimization operation in equation (2.3) may cause extra difficulty in solving it. Motivated by the intuition that total workload in the line should be greater than the line capacity because wafers are released at the maximum capacity $r_{0}$, i.e., assuming $\sum_{j=1}^{J}\left[w_{j}(n)+r_{j-1}(n)\right] \tau_{j} \geq M T$, we first consider the solution to

$$
r_{j}(n)=\frac{w_{j}(n)+r_{j-1}(n)}{\sum_{i=1}^{J}\left[w_{i}(n)+r_{i-1}(n)\right] \tau_{i}} M T \quad \text { for } j=1, \ldots, J
$$

We then check if the solution to equation (3.1) is also a solution to equation (2.3). For notational conciseness, we omit the index $n$ in the later derivations of this section.

We now show that equation (3.1) has a unique solution. Let

$$
\alpha \equiv \frac{M T}{\sum_{j=1}^{J}\left(w_{j}+r_{j-1}\right) \tau_{j}} .
$$

Equation (3.1) ${ }^{\circ}$ can then be rewritten as

$$
r_{j}=\alpha\left(w_{j}+r_{j-1}\right) \equiv g_{j}(\mathbf{r})
$$

or in a matrix form

$$
\mathbf{r}=\mathbf{G}(\mathbf{r}) \text { where } \mathbf{r}=\left[\begin{array}{lll}
r_{1} & \cdots & r_{J}
\end{array}\right]^{\prime} \text { and } \mathbf{G}=\left[\begin{array}{lll}
g_{1} & \cdots & g_{J}
\end{array}\right]^{\prime} .
$$

Let $\mathbf{r}^{*}$ be a solution of equation (3.4). From equation (3.2),

$$
\mathbf{r}^{*}=\alpha^{*}\left[\begin{array}{ccccc}
0 & 0 & \cdots & 0 & 0 \\
1 & 0 & \cdots & 0 & 0 \\
0 & 1 & \cdots & 0 & 0 \\
\vdots & \vdots & \ddots & \vdots & \vdots \\
0 & 0 & 0 & 1 & 0
\end{array}\right] \mathbf{r}^{*}+\alpha^{*}\left[\begin{array}{c}
r_{0}+w_{1} \\
w_{2} \\
w_{3} \\
\vdots \\
w_{J}
\end{array}\right] \text { where } \alpha^{*}=\frac{M T}{\sum_{j=1}^{J}\left(w_{j}+r_{j}^{*}\right) r_{j}}
$$

Expressing $\mathbf{r}^{*}$ in terms of $\alpha^{*}, w_{j}$ for $j=1, \ldots, J$, and $r_{0}$, we have

Equivalently,

$$
\mathbf{r}^{*}=\alpha^{*}\left[\begin{array}{cccc}
1 & 0 & \cdots & 0 \\
\alpha^{*} & 1 & \cdots & 0 \\
\vdots & \vdots & \ddots & 0 \\
\left(\alpha^{*}\right)^{J-1} & \left(\alpha^{*}\right)^{J-2} & \cdots & 1
\end{array}\right]\left[\begin{array}{c}
r_{0}+w_{1} \\
w_{2} \\
\vdots \\
w_{J}
\end{array}\right]
$$

$$
r_{j}^{*}=\left(\alpha^{*}\right)^{j} r_{0}+\sum_{i=1}^{j}\left(\alpha^{*}\right)^{j-i+1} w_{i} \quad \text { for } j=1,2, \ldots,
$$

where both $r_{j}^{*}$ and $\alpha^{*}$ are unknowns. As $\mathbf{r}^{*}$ satisfies equation (3.1) according to our assumption,

$$
\sum_{j=1}^{J} r_{j}^{*} \tau_{j}=M T
$$

Substituting equation (3.5) into (3.6), we obtain a polynomial equation in terms of the unknown $\alpha^{*}$ only :

$$
\sum_{j=1}^{J}\left[\left(\alpha^{*}\right)^{j} r_{0}+\sum_{i=1}^{j}\left(\alpha^{*}\right)^{j-i+1} w_{i}\right] \tau_{j}=M T
$$

Define a polynomiai function

$$
q(\alpha) \equiv \sum_{j=1}^{J}\left[\alpha^{j} r_{0}+\sum_{i=1}^{j} \alpha^{j-i+1} w_{i}\right] \tau_{j}-M T,
$$

where $q(0)=-M T<0$ and $q(1)=\sum_{j=1}^{J} \sum_{i=1}^{j} w_{i} \tau_{j} \geq 0$.

\section{Fact 3.1:}

Since $q(0) q(1) \leq 0$, there exists a root $\alpha^{*} \in(0,1]$. 
Fact 3.2 :

$$
\frac{d}{d \alpha} q(\alpha)=\sum_{j=1}^{J}\left[j \alpha^{j-1} r_{0}+\sum_{i=1}^{j}(j-i+1) \alpha^{j-i} w_{i}\right] \tau_{j}>0 \quad \text { for } \alpha>0
$$

i.e., $q(\alpha)$ is a strictly increasing function for $\alpha>0$.

A lemma can be deduced from the above two facts :

Lemma 3.1:

$q(\alpha)=0$ has a unique root $\alpha^{*}$ for $\alpha>0$ and $\alpha^{*} \in(0,1]$.

Since

$$
\alpha^{*}=\frac{M T}{\sum_{j=1}^{J}\left(w_{j}+r_{j-1}\right) \tau_{j}}<1,
$$

the previous assumption that $\sum_{j=1}^{J}\left(w_{j}+r_{j-1}\right) \tau_{j} \geq M T$ and therefore solving equation (3.1) is equivalent to solving equation (2.3) are proved. In summary, a Theorem can be concluded as follows :

\section{Theorem 1:}

Given an initial WIP distribution $\left\{w_{j}(n) \geq 0, j=1,2, \ldots, J\right\}$ and a constant release rate $r_{0}$ defined by equation (2.1), equation (2.3) has a unique solution $\mathbf{r}^{*} \geq \mathbf{0}$ that fully allocates the machine capacity with

$$
r_{j}^{*}(n)=\left[\alpha^{*}(n)\right]^{j} r_{0}+\sum_{i=1}^{j}\left[\alpha^{*}(n)\right]^{j-i+1} w_{i}(n)
$$

where $\alpha^{*}(n)$ is the unique solution to and $0<\alpha^{*}(n) \leq 1$.

$$
\sum_{j=1}^{J}\left[\alpha^{j}(n) r_{0}+\sum_{i=1}^{j} \alpha^{j-i+1}(n) w_{i}(n)\right] \tau_{j}-M T=0, \quad \text { for } \alpha(n)>0,
$$

\section{Line Balancing Effect}

In this section, it is shown that the reentrant line can be balanced by repetitive application of the proportional machine allocation scheme over time. In the following discussions, we denote $\mathbf{r}^{*}(n)$ as $\mathbf{r}(n)$ and $\alpha^{*}(n)$ as $\alpha(n)$ for notational simplicity.

\section{IV.I Some Basics}

The flow balance equation between two stages is

which can be written, by using (3.3) and (3.10), as

$$
w_{j}(n+1)=w_{j}(n)-r_{j}(n)+r_{j-1}(n),
$$

$$
w_{j}(n+1)=[1-\alpha(n)]\left[\sum_{i=1}^{j} \alpha^{j-i}(n) w_{i}(n)+\alpha^{j-1}(n) r_{0}\right], \quad \text { for } j=1, \ldots,
$$

or in a matrix form as

$$
\mathbf{w}(n+1)=(1-\alpha(n))\left[\begin{array}{cccc}
1 & 0 & \cdots & 0 \\
\alpha(n) & 1 & \cdots & 0 \\
\vdots & \vdots & \ddots & \vdots \\
\alpha^{J-1}(n) & \alpha^{J-2}(n) & \cdots & 1
\end{array}\right] \mathbf{w}(n)+(1-\alpha(n))\left[\begin{array}{c}
1 \\
\alpha(n) \\
\vdots \\
\alpha^{J-1}(n)
\end{array}\right] r_{0}
$$


To eliminate $r_{0}$ from the expression of equation (4.3), we rewrite equation (3.2) and obtain

From equation (3.10),

$$
\sum_{j=1}^{J}\left[w_{j}(n)+r_{j-1}(n)\right] \tau_{j}=\frac{M T}{\alpha(n)} .
$$

$$
\begin{aligned}
& \sum_{j=1}^{J}\left[w_{j}(n)+r_{j-1}(n)\right] \tau_{j} \\
= & \sum_{j=1}^{J} w_{j}(n) \tau_{j}+\sum_{j=2}^{J} \sum_{i=1}^{j-1} \alpha^{j-i}(n) w_{i}(n) \dot{\tau}_{j}+r_{0} \sum_{j=1}^{J} \alpha^{j-1}(n) \tau_{j}
\end{aligned}
$$

and from the definition of $r_{0}$,

$$
\frac{M T}{\alpha(n)}=\frac{r_{0} \sum_{j=1}^{J} \tau_{j}}{\alpha(n)} .
$$

By combining (4.4), (4.5.1), and (4.5.2), the constant wafer release rate $r_{0}$ can be represented in $\alpha(n),\left\{\tau_{j}, j=1, \ldots, J\right\}$ and $\mathbf{w}(n)$ :

and equation (4.3) becomes

$$
r_{0}=\frac{\alpha(n)}{\sum_{j=1}^{J}\left[1-\alpha^{j}(n)\right] \tau_{j}} \sum_{j=1}^{J}\left[w_{j}(n) \sum_{i=j}^{J} \alpha^{i-j}(n) \tau_{i}\right],
$$

$$
\begin{aligned}
& \text { with } \mathbf{A}(n)=(1-\alpha(n))\left[\begin{array}{cccc}
\mathbf{w}(n+1)=\mathbf{A}(n) \mathbf{w}(n) \\
1 & 0 & \cdots & 0 \\
\alpha(n) & 1 & \cdots & 0 \\
\vdots & \vdots & \ddots & \vdots \\
\alpha^{J-1}(n) & \alpha^{J-2}(n) & \cdots & 1
\end{array}\right] \\
& +\frac{\alpha(n)[1-\alpha(n)]}{\sum_{j=1}^{J}[1-\alpha j(n)] \tau_{j}}\left[\begin{array}{cccc}
f_{1(n)} & f_{2}(n) & \cdots & f_{J}(n) \\
\alpha(n) f_{1}(n) & \alpha(n) f_{2}(n) & \cdots & \alpha(n) f_{J}(n) \\
\vdots & \vdots & \vdots & \vdots \\
\alpha^{J-1}(n) f_{1(n)} & \alpha^{J-1}(n) f_{2}(n) & \cdots & \alpha^{J-1}(n) f_{J}(n)
\end{array}\right]
\end{aligned}
$$

where $f_{j}(n)=\sum_{i=j}^{J} \alpha^{i-j}(n) \tau_{i}$ for $j=1, \ldots$.

To analyze the line balancing effect of the proportional machine allocation algorithm, let us first introduce the following proposition of [MaO79].

Proposition:

Let $\mathbf{p}=\left[\begin{array}{lll}p_{1} & \cdots & p_{n}\end{array}\right]^{\prime}$ and $\mathbf{q}=\left[\begin{array}{lll}q_{1} & \cdots & q_{m}\end{array}\right]^{\prime}$ be two fixed vectors with nonnegative components such that $\sum_{i=1}^{n} p_{i}=\sum_{j=1}^{m} q_{j}=1$. For $\mathbf{x} \in R^{n}$ and $\mathbf{y} \in R^{m}$, the inequality $\sum_{i=1}^{n} p_{i} g\left(x_{i}\right) \leq \sum_{j=1}^{m} q_{j} g\left(y_{j}\right)$ holds for all continuous convex function $g: R \rightarrow R$, if and only if there exists a matrix $\mathbf{A}=\left[a_{i j}\right]_{n \times m}$ with the properties (1) $a_{i j} \geq 0$, for all i, j, (2) $\mathbf{A e}=\mathbf{e}$, where $\mathbf{e}=\left[\begin{array}{lll}1 & \cdots & 1\end{array}\right]^{\prime}$ and (3) $\mathbf{p}^{\prime} \mathbf{A}=\mathbf{q}^{\prime}$ such that $\mathbf{x}=\mathbf{A y}$. The strict inequality holds if $g: R \rightarrow R$ is strictly convex.

\section{IV.II Proof of Line Balancing}

Now, suppose that the reentrant line reaches balance, i.e., $\lim _{n \rightarrow \infty} r_{j}(\dot{n})=r_{0}$, for $j=1, \ldots, J$, then it follows from equation (3.3) that $\lim _{n \rightarrow \infty} w_{j}(n)=w^{*} \geq 0$. 
Lemma 4.1:

The balanced line has a uniform WIP distribution over all the stages at a level $w^{*}=\sum_{j=1}^{J} p_{j} w_{j}(0)$, where $p_{j}=\left(\sum_{i=j}^{J} \tau_{i}\right) /\left(\sum_{j=1}^{J} \sum_{i=j}^{J} \tau_{i}\right)$.

Proof:

Note that $\sum_{i=j}^{J} \tau_{i}$ is the residual processing time (processing time to finish) of a wafer. Define the total residual processing time requirement $L(n)$ of the line with WIP distribution $\mathbf{w}(n)$ at the beginning of the nth time period as

$$
L(n) \equiv \sum_{j=1}^{J} w_{j}(n) \sum_{i=j}^{J} \tau_{i}
$$

The total residual processing time requirement of the whole line between two time periods then have the relation

$$
L(n+1)=L(n)+r_{0} \sum_{j=1}^{J} \tau_{j}-\sum_{j=1}^{J} r_{j}(n) \tau_{j} \quad \text { for } n=0,1,2, \ldots,
$$

with $L(0)=\sum_{j=1}^{J} w_{j}(0) \tau_{j}$. Since the workload released to the line in each time period equals to the machine hours that can be provided by the machine group and the machine hours are always utilized completely, i.e.,

it can be easily derived that

$$
r_{0} \sum_{j=1}^{J} \tau_{j}=M T=\sum_{j=1}^{J} r_{j}(n) \tau_{j},
$$

$$
L(n)=\sum_{j=1}^{J} w_{j}(0) \sum_{i=j}^{J} \tau_{i}, \text { for } n=1,2, \ldots
$$

When the line is balanced,

and therefore

$$
\sum_{j=1}^{J} w^{*} \sum_{i=j}^{J} \tau_{i}=\sum_{j=1}^{J} w_{j}(0) \sum_{i=j}^{J} \tau_{i}
$$

$$
w^{*}=\left[\sum_{j=1}^{J} w_{j}(0) \sum_{i=j}^{J} \tau_{i}\right] /\left[\sum_{j=1}^{J} \sum_{i=j}^{J} \tau_{i}\right] . \quad \text { Q.E.D. }
$$

Now, let us prove that repetitive application of the proportional machine allocation scheme leads to line balance. Consider a Lyapunov function defined as

$$
V(\mathbf{w}(n))=\sum_{j=1}^{J} p_{j}\left[w_{j}(n)-w^{*}\right]^{2} \text {. }
$$

If the parameter $p_{j}$ is interpreted as a normalized residual processing time of a wafer in the jth buffer, the Lyapunov function then has the meaning of weighted least square deviation of the line WIP distribution from the balance distribution.

Let $\mathbf{p}=\mathbf{q}=\left[\begin{array}{lll}p_{1} & \cdots & p_{J}\end{array}\right]^{\prime}$. We shall show that $\mathbf{A}(n), \quad \mathbf{p}$ and $g\left(w_{j}(n)\right)=\left[w_{j}(n)-w^{*}\right]^{2} \quad$ satisfy the conditions of the Proposition [MaO79] and $V(\mathbf{w}(n+1))<V(\mathbf{w}(n))$ when $\mathbf{w}(n) \neq \mathbf{w}^{*}$. This implies that $\lim _{n \rightarrow \infty} w_{j}(n)=w^{*}$ for $j=1, \ldots, J$, that is, the repetitive application of the proportional machine allocation scheme results in line balancing eventually.

Lemma 4.2:

(1) $\sum_{j=1}^{J} p_{j}=\sum_{j=1}^{J} q_{j}=1$;

(2) $a_{i j} \geq 0$

(3) $\mathbf{A}(n) \mathbf{e}=\mathbf{e}$;

(4) $\mathbf{p}^{\prime} \mathbf{A}(n)=\mathbf{p}^{\prime}$. 
Proof :

(1) It is obvious by definition.

(2) It is true because $0<\alpha(n) \leq 1$.

(3) the ith row of $\mathbf{A}(n) \mathbf{e}$

$$
\begin{aligned}
& =[1-\alpha(n)]\left[1+\alpha(n)+\cdots+\alpha^{J-1}(n)\right] \\
& +\left(\frac{\alpha(n)[1-\alpha(n)]}{\sum_{j=1}^{J}\left[1-\alpha^{j}(n)\right] \tau_{j}}\right) \alpha^{i-1}(n)\left[f_{1}(n)+f_{2}(n)+\cdots+f_{J}(n)\right] \\
& =1-\alpha^{i}(n)+\left(\frac{\alpha^{i}(n)}{\sum_{j=1}^{J}\left[1-\alpha^{j}(n)\right] \tau_{j}}\right)\left\{[1-\alpha(n)] \tau_{1}+\left[1-\alpha^{2}(n)\right] \tau_{2}+\cdots+\left[1-\alpha^{J}(n)\right] \tau_{J}\right\} \\
& =1
\end{aligned}
$$

(4) the ith column of $\mathbf{p}^{\prime} \mathbf{A}(n)$

$$
\begin{aligned}
& =[1-\alpha(n)]\left[p_{i}+\alpha(n) p_{i+1}+\cdots+\alpha^{J-i}(n) p_{J}\right] \\
& +\left\{[\alpha(n)[1-\alpha(n)]] /\left[\sum_{j=1}^{J}\left[1-\alpha^{j}(n)\right] \tau_{j}\right]\right\}\left[p_{1}+\alpha(n) p_{2}+\cdots+\alpha^{J-1}(n) p_{J}\right] f_{i}(n) \\
& =\frac{[1-\alpha(n)] \tau_{i}+\left[1-\alpha^{2}(n)\right] \tau_{i+1}+\cdots+\left[1-\alpha^{J-i+1}(n)\right] \tau_{J}}{\sum_{j=1}^{J} \sum_{i=j}^{J} \tau_{i}}+\frac{\alpha(n)\left[\tau_{i}+\alpha(n) \tau_{i+1}+\cdots+\alpha^{J-i}(n) \tau_{J}\right]}{\sum_{j=1}^{J} \sum_{i=j}^{J} \tau_{i}} \\
& =\left(\sum_{i=j}^{J} \tau_{i}\right) /\left(\sum_{j=1}^{J} \sum_{i=j}^{J} \tau_{i}\right)
\end{aligned}
$$$$
=p_{i}
$$

Q.E.D

\section{Lemma 4.3:}

$0 \leq V(\mathbf{w}(n+1))<V(\mathbf{w}(n)) \quad$ if $\mathbf{w}(n) \neq \mathbf{w}^{*}$ and $V\left(\mathbf{w}^{*}\right)=0$. Proof:

It is straight forward by the fact that $g\left(w_{j}(n)\right)=\left[w_{j}(n)-w^{*}\right]^{2}$ is continuous and strictly convex and by using the Proposition of [MaO79].

Q.E.D.

In summary of Lemmas 4.1 to 4.3 , we arrive at the following theorem :

\section{Theorem 2 : (Line Balancing Effect)}

Given an arbitrarily initial WIP distribution $\left\{w_{j}(0), j=1, \ldots, f\right\}$ and a line capacity $M T$, if $r_{0}=(M T) /\left(\sum_{j=1}^{J} \tau_{j}\right)$, the repetitive application of the proportional machine allocation scheme (i.e., equation (2.3)) over time results in
(a) Linear Output $\lim _{n \rightarrow \infty} r_{j}(n)=r_{0} \quad$ for $j=1, \ldots$, .
(b) Uniform WIP Distribution $\lim _{n \rightarrow \infty} w_{j}(n)=w^{*}=\sum_{j=1}^{J} p_{j} w_{j}(0)$ for $j=1, \ldots$, .

\section{Conclusions}

In this paper, a proportional machine allocation scheme was analyzed for a reentrant production line of single product type and single machine group. It was shown that given an initial WIP distribution and a constant wafer release rate of maximum long term average line capacity, there exists a unique full-capacity machine allocation for the line and that the whole reentrant line can be balanced by repetitive application of the proportional 
machine allocation scheme. Generalization of this analysis to multiple product types and multiple machine groups has been under further study.

\section{References}

[AtD86] R. W. Atherton, J. E. Dayhoff, "Signature Analysis : Simulation of Inventory, Cycle Time, and Throughput Trade-offs in Wafer Fabrication", IEEE Trans. On. Comp. Hybr. and Manuf., Vol. CHMT-9, No. 4, 1986, pp.498-507.

[BiT88] G. R. Bitran and D. Tirupati, "Planning and Scheduling for Epitaxial Wafer Production Facility", Operation Research, Vol. 36, No. 3, May-June 1988, pp. 377-395.

[BSG90] S. X. Bai, N. Srivatsan and S. G. Gershwin, "Proceedings of the 9th IEEE International Electronics Manufacturing Technology Symposium", Washington, D. C., October 1990.

[CFY92] D. Connors, G. Feigin and D. Yao, "Scheduling Semiconductor Lines Using a Fluid Network Model", Proceedings of the 3rd International Conference on Computer Integrated Manufacturing, Troy, New York, May 1992, pp. 174-183.

[CLP92] S. C. Chang, L. H. Lee, L. S. Pang, Y. C. Chang, P. C. Lin, T. W. Y. Chen, " Daily Target Generation and Machine Allocation for Integrated Circuit Fabrication", The Second International Conference on Automatic Technology, Vol. 4, Taipei, July 1992, pp.47-52.

[Kum93] P. R. Kumar, "Reentrant Lines", Queueing Systems : Theory and Application, Vol. 13, pp. 87-110, 1993.

[LoG88] C. Lozinski, C. R. Glassey, "Bottleneck Starvation Indicators for Shop Floor Control", IEEE Transactions on Semiconductor Manufacturing, Vol. 1, No. 4, November 1988, pp. 147-153.

[LRK93] S. C. H. Lu, D. Ramaswamy, P. R. Kumar, "Efficient Scheduling Policies to Reduce Mean and Variance of Cycle-Time in Semiconductor Manufacturing Plants", IEEE Transactions on Semiconductor Manufacturing, Vol. 7, No. 3, pp.374-388.

[LuK91] S. H. Lu, P.R. Kumar, "Distributed Scheduling Based on Due Dates and Buffer Priorities", IEEE Transactions on Automatic Control, Vol. 36, No. 12, December 1991, pp. 1406-1416.

[MaO79] A. W. Marshall, I. Olkin, Inequalities : Theory of Majorization and Its Application, Mathematics in Science and Engineering, Vol. 143, New York, 1979, pp. 417-418.

[PeK94] J. R. Perkins, P. R. Kumar, "Optimal Control of Pull Manufacturing System", Research Report, ECE Dept., Univ. of Illinois, Urbana, IL, 1994.

[Wei88] L. M. Wein, "Scheduling Semiconductor Wafer Fabrication", IEEE Transactions on Semiconductor Mamufacturing, Vol. 1, No. 3, August 1988, pp. $115-130$. 